Notre Dame Journal of Formal Logic

Volume 21, Number 3, July 1980

\title{
Nominalism and Conceptualism as Predicative Second-Order Theories of Predication
}

\author{
NINO COCCHIARELLA
}

There appears to be a growing consensus, even if not unanimity, that standard predicative second-order logic is the appropriate logical medium for the representation of a nominalist theory of predication.* We agree that this is indeed the case and formulate in this paper a model-theoretic approach which justifies that claim. ${ }^{1}$ Because it is model-theoretic, our approach differs from the truth-value semantics approach of Leblanc and Weaver. ${ }^{2}$ Amongst other reasons, we prefer our model-theoretic approach so as to accommodate those nominalists for whom the assumption that there are potentially as many names as there are individuals is not acceptable.

The models involved in our semantics, moreover, are precisely the same models as are already involved in standard first-order logic. Assignments of values (drawn from the domain of a given model) to the individual variables are extended, however, to what, relative to a given first-order language, we call nominalistic assignments to the $n$-place predicate variables (for each positive integer $n$ ): these assign first-order formulas (wffs) of the language in question, relative to the free occurrences of $n$ distinct individual variables occurring in those wffs, to the $n$-place predicate variables. The satisfaction by such an assignment of a second-order wff in a model is then defined by a double recursion on the logical structure of the wff and on the number of nested predicate quantifiers occurring therein.

\footnotetext{
*The author is indebted to the National Endowment for the Humanities for support during the research and writing of this paper.
} 
It is natural of course that a first-order wff, relative to $n$ individual variables occurring therein as argument indicators, should be understood as representing an $n$-place predicate expression of the language in question; and in fact in an applied first-order theory based upon that language such a firstorder wff would constitute the definiens of a possible definition for an $n$-place predicate constant not already belonging to that language or occurring in that theory. Potentially, of course, there are infinitely many predicate constants that might be introduced into a first-order theory in this way; and it is just over such a potential infinity, and no more, that our predicate quantifiers, nominalistically interpreted, are understood to range when we turn to the predicative second-order counterpart of a given first-order theory.

Finally, in order to better understand the implicit background of our nominalistic semantics, we include in a final section of this paper a brief comparison of nominalism, as represented by standard predicative second-order logic, with a closely related form of conceptualism, represented by a certain nonstandard predicative second-order logic formulated by the author in an earlier paper.

\section{Nominalism and the problem of universals As a theory of predication,} nominalism is concerned with the problem of universals, where by a universal we mean that which is of such a nature as can be predicated of many things (cf. Aristotle, De Interpretatione, 17a39). Traditionally, there have been three general types of approaches to the problem of universals: nominalism, conceptualism, and realism, with nominalism being the most restrictive. In the context of a theory of predication, we understand the problem of universals to be the problem of explaining their predicable nature, i.e., that in which their universality consists.

Now the general thesis of nominalism is that universals have only a formal mode of existence, i.e., that beyond the predicate expressions that occur or can occur in language there are no universals. In this regard we understand nominalism as claiming that universals are none other than predicate expressions and that their predicable nature, in which consists their universality, is none other than the logico-grammatical role they are represented as having in the logical forms of standard first-order predicate logic. Semantically, this role includes the fact that predicate expressions can be said to be either true or false of individuals.

In addition to there being no universals beyond predicate expressions, a second general thesis of nominalism is that there are only individuals (in the logical sense), i.e., that quantificational reference is univocal and applies only to the individual things of which our various predicate expressions may be said to be either true or false. Accordingly, the referential sense in which there are universals according to nominalism is but a restricted form of the sense in which there are individuals. However, unlike other individuals, quantificational reference to nominalistic universals requires the medium of a metalanguage for its expression, and a theory of predication in terms of such reference must therefore also be metalinguistic. In this respect nominalism differs from both conceptualism and realism in that the theories of predication associated with the latter can be formulated as pure theories of logical form 
in which, in particular, descriptive constants of the metalanguage need not occur. As the basis for a theory of logical form, it is preferable that we distinguish a theory of predication from its applications to particular domains of discourse, including in this regard even the domain of the predicate expressions of a collection of object languages.

Fortunately there is another, albeit nonreferential, notion of quantification which nominalism may allow with respect to the role of predicate expressions, i.e., which pertains to their predicable nature alone, and which allows for the formulation of a pure theory of logical form. This is the so-called substitution interpretation of predicate quantifiers whereby the significance of such a quantifier in the context of an applied first-order theory is exhausted by the totality of its substituends, i.e., predicate expressions in the form of open wffs of that theory, rather than by a reference to real universals existing independently of language and supplemental to the domain of discourse of the theory. In this regard nominalism can be developed as a second-order theory of predication, i.e., as one which allows quantification with respect to predicate as well as subject positions, even if the significance of predicate quantifiers, unlike those that reach into subject positions and bind individual variables, is not strictly referential. There will, however, be certain constraints which such an interpretation imposes upon the specification of universals in a nominalistic theory of predication. These constraints, as we show below, are in fact precisely those involved in the comprehension principle of standard predicative second-order logic.

2 First-order theories Because they take quantificational reference to be univocal and only with respect to the individual things of which our various predicate expressions may be true or false, nominalists have restricted themselves to first-order theories when dealing with particular domains of discourse. Even free predicate variables are not allowed to occur as part of the object languages of these first-order theories but are viewed instead as dummy schema letters of the metalanguage. (Thus, in this regard, the proper axioms and theorems of a given first-order theory are distinguished from its axiom and theorem schemata.) And the restriction is in fact quite justified, given the nominalist standpoint, since, as it turns out, the predicative second-order counterpart of any first-order theory is a conservative extension of that firstorder theory, i.e., every first-order wff which is provable in the predicative second-order counterpart of that first-order theory is already provable in the first-order theory itself. Nevertheless, this predicative second-order theory, we maintain, contains the more appropriate representative of the nominalist's background theory of logical form and implicit theory of predication, particularly since it encapsulates in a single system all of the possible, and potentially infinitely many, definitional extensions of the first-order theory without actually introducing or considering collectively as an actual infinity all of the potential predicates that might be introduced in such extensions. ${ }^{3}$

We shall for convenience view every first-order theory as having the same primitive logical constants, which we take to be $\sim$, the negation sign, $\rightarrow$, the (material) conditional sign, $\forall$, the universal quantifier, and $=$, the identity sign. Other logical constants are understood to be defined in the usual way (as 
abbreviatory devices of the metalanguage). We shall also use parentheses and brackets as auxiliary signs. We use ' $x$ ' and ' $y$ ', with or without numerical subscripts, to refer to individual variables, and ' $P^{n}$ ', with or without subscripts, to refer to $n$-place predicate constants. (For simplicity of presentation we assume individual and functor constants to have been eliminated in favor of predicates alone.)

Since all our first-order languages have the same logical constants and auxiliary signs, they differ only in what predicate constants they contain. We can therefore identify such a language directly with the set of its predicate constants. That is, henceforth we understand a language $L$ to be a set of predicate constants. The atomic $w f f s$ of $L$ then are all expressions of the form $(x=y)$ or $P^{n}\left(x_{1}, \ldots, x_{n}\right)$, where $P^{n} \in L$. The set of first-order $w f f s$ of $L$ is then determined inductively as the smallest set $K$ containing every atomic wff of $L$ and such that $\sim \varphi,(\varphi \rightarrow \psi),(\forall x) \varphi \in K$, whenever $\varphi, \psi \in K$ and $x$ is an individual variable. We shall use ' $\varphi$ ', ' $\psi$ ', ' $\chi$ ' to refer to wffs (first-order or otherwise), and we understand the bondage and freedom (of occurrences) of individual variables in wffs to be defined in the usual way. We take $\varphi(x / y)$ to be the result of replacing each free occurrence of $y$ in $\varphi$ by a free occurrence of $x$, if such a result exists, and otherwise we take it to be just $\varphi$ itself. Similarly, we understand $\varphi\left(x_{1} / y_{1}, \ldots, x_{n} / y_{n}\right)$ to be the result of simultaneously replacing each free occurrence of $y_{1}, \ldots, y_{n}$ by free occurrences of $x_{1}, \ldots, x_{n}$, respectively.

Ordinarily, we take a first-order theory $T$ to be a pair $\langle A, L\rangle$, where $L$ is a language and $A$ is a set of first-order wffs of $L$, each of which is understood to be an axiom of $T$. A definitional extension of $T$ then is a pair $\langle A \cup\{D\}$, $L \cup\left\{P^{n}\right\}$, where $D$ is a possible definition of $P^{n}$ in terms of $L$. However, since we shall actually be bypassing the definitional extensions of first-order theories by resorting to their predicative second-order counterparts instead, we can for convenience identify a first-order theory directly with its axiom set. The language of such a theory is then the set of predicate constants occurring in these axioms.

Where $T$ is a first-order theory and $\varphi$ is a first-order wff of the language of $T$, we understand $\varphi$ to be a theorem of $T$, in symbols $\leftarrow_{T} \varphi$ iff $T \vdash \varphi$, i.e., iff $\varphi$ is derivable from $T$ in standard first-order predicate logic (with identity), i.e., iff $\varphi$ is the terminal wff of a finite sequence of first-order wffs of the language of $T$ every constituent of which is either a member of $T$, a logical axiom, or is obtained from preceding constituents by modus ponens.

By a logical axiom in the above definition we mean any generalization ${ }^{4}$ of a tautologous wff or of any wff of one of the following forms:

$$
(\forall x)(\varphi \rightarrow \psi) \rightarrow((\forall x) \varphi \rightarrow(\forall x) \psi)
$$

$\varphi \rightarrow(\forall x) \varphi$, where $x$ does not occur free in $\varphi$

$(\exists y)(x=y)$, where $x, y$ are distinct variables

$x=y \rightarrow(\varphi \rightarrow \psi)$, where $\varphi$ is an atomic wff and $\psi$ is obtained from $\varphi$ by replacing an occurrence of $y$ by an occurrence of $x$.

It is well-known that the above substitution-free characterization of logical axioms is equivalent to the more usual axiomatic formulations of standard predicate logic with identity and that both Leibniz's law: 
LL $\quad x=y \rightarrow(\varphi \leftrightarrow \psi)$

where $\psi$ is obtained from $\varphi$ by replacing one or more free occurrences of $y$ in $\varphi$ by a free occurrence of $x$, and the principle of universal instantiation:

UI $\quad(\forall x) \varphi \rightarrow \varphi(y / x)$

are provable (cf. [6] and [11]).

3 Referential semantics of first-order theories Though universals have only a formal mode of existence according to nominalism, individuals (in the logical sense) nevertheless are presumed to be fully real independent entities. Individuals may indeed be only concrete particulars, as they are usually understood on more traditional accounts, but, at least on modern accounts, they may also include abstract entities, such as sets or real numbers, as well. (Quine is a prime example of such a modern nominalist.) The basic theses of nominalism, after all, are that there are no universals beyond predicate expressions themselves and that there are only individuals, whether these be concrete or abstract. And we have added to these only the explanatory or clarifying thesis that the predicable nature of nominalistic universals, i.e., of possible predicate expressions, is the logico-grammatical role they are represented as having in the logical forms of standard first-order predicate logic (with identity). Accordingly, it is inappropriate that we require the nominalist to assume that there are potentially as many names as there are individuals and that a substitutional interpretation of quantifiers reaching into subject positions can be used in his theory of predication instead of the objective interpretation of a quantificational reference to individuals. For this reason we take a referential semantics (of first-order wffs), such as that abstractly represented in model theory, as being more appropriate to nominalism. ${ }^{5}$

Accordingly, where $L$ is a language, we take $थ$ to be an $L$-model iff $\mathscr{\imath}=$ $\langle D, f\rangle$, for some nonempty set $D$ and $L$-indexed set $f$ such that for all $P^{n} \in L$, $f\left(P^{n}\right) \subseteq D^{n}$. Naturally, we take $D$ to be the domain of discourse of the model and we identify all the ( $n$-tuples of) individuals in $D$ that a predicate constant $P^{n}$ of $L$ is true of by means of their membership in $f\left(P^{n}\right)$. We understand a (first-order) referential assignment in 2 to be a function $A$ whose domain is included in the set of individual variables and such that $A(x) \epsilon D$, for each individual variable $x$. (Where $d \in D$, we set $A(d / x)=(A-\{\langle x, A(x)\rangle\}) \cup\{\langle x, d\rangle\}$.)

We extend the notion of when a predicate of $L$ is true of an ( $n$-tuple of) individual(s) in 2 . to arbitrary open wffs (predicate expressions) of $L$ by recursively defining the conditions under which a wff of $L$ is satisfied by a referential assignment $A$ in $\mathscr{Q}$ as follows:

1. $A$ satisfies $(x=y)$ in 2 iff $A(x)=A(y)$

2. $A$ satisfies $P^{n}\left(x_{1}, \ldots, x_{n}\right)$ in 2 . iff $\left\langle A\left(x_{1}\right), \ldots, A\left(x_{n}\right)\right\rangle \in f\left(P^{n}\right)$

3. A satisfies $\sim \varphi$ in $\mathscr{A}$ iff $A$ does not satisfy $\varphi$ in $\mathscr{A}$

4. A satisfies $(\varphi \rightarrow \psi)$ in $\mathscr{U}$ iff either $\mathscr{U}$ does not satisfy $\varphi$ in $\mathscr{U}$ or $A$ satisfies $\psi$ in 2 ; and

5. A satisfies $(\forall x) \varphi$ in 2 . iff for all $d \in D, A(d / x)$ satisfies $\varphi$ in $\mathscr{x}$. 
We say then that a wff $\varphi$ is a (first-order) logical consequence of a set $T$ of wffs, in symbols $T \vDash \varphi$, iff for every language $L$ such that $T \cup\{\varphi\}$ is a set of wffs of $L$ and for every $L$-model $\mathfrak{A}$ and every referential assignment $A$ in $\mathscr{A}$, if $A$ satisfies every member of $T$ in $\mathscr{A}$, then $A$ satisfies $\varphi$ in $\mathscr{R}$. It is well-known of course that (first-order) logical consequence and derivability within standard first-order predicate logic (with identity) coincide.

Completeness theorem If $T$ is a first-order theory and $\varphi$ is a wff of the language of $T$, then $T \vdash \varphi$ iff $T \vDash \varphi$.

4 Standard predicative second-order logic We turn now to a characterization of the logical background of the predicative second-order counterparts of first-order theories. We assume for this purpose the availability, for each natural number $n$, of denumerably many $n$-place predicate variables. We shall use ' $F^{n}$ ' and ' $G$ ', with or without numerical subscripts, to refer to $n$-place predicate variables, though we shall occasionally drop the superscript whenever the context makes clear the degree of the predicate in question.

When $L$ is a language, the atomic wffs of $L$ are defined as before except that now $F^{n}\left(x_{1}, \ldots, x_{n}\right)$ is also an atomic wff of $L$, for all $n$-place predicate variables $F^{n}$ and individual variables $x_{1}, \ldots, x_{n} \cdot{ }^{6}$ (It should be noted, however, that now not every atomic wff is a first-order wff since, by definition, no predicate variable occurs in a first-order wff.) The set of (second-order) $w f f s$ of $L$ is determined inductively as the smallest set $K$ containing all the atomic wffs of $L$ (in the newly extended sense) and such that $\sim \varphi,(\varphi \rightarrow \psi),(\forall x) \varphi$, $\left(\forall F^{n}\right) \varphi \in K$, whenever $\varphi, \psi \in K, x$ is an individual variable, $n \in \omega$, and $F^{n}$ is an $n$-place predicate variable. (Of course every first-order wff is a second-order wff, but not conversely.)

We understand the bondage and freedom of (occurrences of) predicate and individual variables in (second-order) wffs to be defined in the usual way; and we take $\varphi\left(P^{n} / F^{n}\right)$ to be the result of replacing each free occurrence of $F^{n}$ in $\varphi$ by a free occurrence of $P^{n}$. Similarly, we assume that the notion of the substitution of a (second-order) wff $\varphi$, relative to $x_{1}, \ldots, x_{n}$ as argument indicators, for an $n$-place predicate variable $F^{n}$ in a (second-order) wff $\psi$, or in symbols:

$$
\breve{\complement}_{\varphi} F\left(x_{1}, \ldots, x_{n}\right) \psi \mid,
$$

to have been defined in the usual way (cf. [1], p. 192).

By a logical axiom we now understand any generalization ${ }^{7}$ of a secondorder wff which is a logical axiom as defined above for first-order theories as well as any generalization of a wff of one of the following forms:

$$
\begin{aligned}
\left(\forall F^{n}\right)(\varphi \rightarrow \psi) & \rightarrow\left(\left(\forall F^{n}\right) \varphi \rightarrow\left(\forall F^{n}\right) \psi\right) \\
\varphi \rightarrow\left(\forall F^{n}\right) \varphi, & \text { where } F^{n} \text { does not occur free in } \varphi \\
\left(\exists F^{n}\right)\left(\forall x_{1}\right) & \ldots\left(\forall x_{n}\right)\left[F\left(x_{1}, \ldots, x_{n}\right) \leftrightarrow \varphi\right] \\
& \text { where } \varphi \text { is a (second-order) wff in which } F^{n} \text { does not occur } \\
& \text { and in which no predicate variable has a bound occurrence, } \\
& \text { and where } x_{1}, \ldots, x_{n} \text { are among the distinct individual vari- } \\
& \text { ables occurring free in } \varphi .
\end{aligned}
$$


We note that on the basis of this substitution-free characterization of logical axioms the following second-order analogue of Leibniz's law is provable:

$$
\mathbf{L L}_{2} \quad\left(\forall x_{1}\right) \ldots\left(\forall x_{n}\right)\left[F\left(x_{1}, \ldots, x_{n}\right) \leftrightarrow \varphi\right] \rightarrow\left[\psi \leftrightarrow \zeta_{\varphi}^{F\left(x_{1}, \ldots, x_{n}\right)} \psi \mid\right] .
$$

(The proof is straightforward by induction on the subwffs of $\psi$. If $\psi$ is atomic, $\mathrm{LL}_{2}$ follows by the first-order UI applied $n$ times, and otherwise it follows by the inductive hypothesis and elementary transformations together with the fact that the substitution is voided (in which case $\mathrm{LL}_{2}$ is tautologous) if $\psi$ begins with a universally quantified variable (other than $x_{1}, \ldots, x_{n}$ ) which occurs free in $\varphi$.) And from $\mathrm{LL}_{2}$ and the comprehension principle, i.e., the last of the above axiom schemes, the second-order principle of universal instantiation is provable:

$$
\mathbf{U I}_{2} \quad\left(\forall F^{n}\right) \psi \rightarrow \breve{S}_{\varphi}^{F\left(x_{1}, \ldots, x_{n}\right)} \psi
$$

where $\varphi$ is a (second-order) wff in which no predicate variable has a bound occurrence, and where $x_{1}, \ldots, x_{n}$ are among the distinct individual variables occurring free in $\varphi .^{8}$ (If $F^{n}$ does not occur in $\varphi$, the proof is by tautologous transformations of $\mathrm{LL}_{2}$, universal generalization, and an obvious use of each of the above axioms for predicate quantifiers. This qualified form of $\mathrm{UI}_{2}$ suffices to prove the rewrite law for bound predicate variables, since where $\varphi$ is $G\left(x_{1}, \ldots, x_{n}\right)$, we have $\left(\forall F^{n}\right) \psi \rightarrow\left(\forall G^{n}\right) \psi(G / F)$ as provable on the basis of the qualified form. And given the rewrite law the qualification that $F^{n}$ not occur in $\varphi$ can be dropped.)

We could of course have taken $\mathrm{UI}_{2}$ instead of the comprehension principle as one of our axiom schemes. The two principles, in other words, are equivalent relative to the remaining axioms. Each in its way specifies the conditions under which a wff $\varphi$, relative to certain of its free individual variables as argument indicators, may be said to determine a value of the bound $n$-place predicate variables. We prefer taking the comprehension principle as basic both because it allows for a substitution-free characterization in which the complex notion of substitution (in all its forms) is not required, and because it states in effect the general conditions under which explicit definitions of predicates are possible. (The free individual and predicate variables occurring in an instance of the comprehension principle serve in effect as parameters for individuals and first-order wffs, respectively.)

Now by a predicative second-order theory we mean any set of secondorder wffs. Thus every first-order theory is a predicative second-order theory as well. The difference is that the logical background now includes the logical axioms of standard predicative second-order logic as well. Where $T$ is a secondorder theory and $\varphi$ is a (second-order) wff of the language of $T$, we understand $\varphi$ to be a theorem of $T$, in symbols $T_{T !} \varphi$, iff $\varphi$ is derivable from $T$ in standard predicative second-order logic (with identity), i.e., iff $\varphi$ is the terminal wff of a finite sequence of (second-order) wffs of the language of $T$ every constituent of which is either a member of $T$, a logical axiom, or is obtained from preceding constituents by modus ponens. Though it is not obvious at this point, it is a consequence of our completeness theorem for standard predicative second- 
order logic that if $T$ is a first-order theory and $\varphi$ is a first-order wff of the language of $T$, then $\bar{T}_{T} \varphi$ iff $\hbar_{T} \varphi$, i.e., the predicative second-order counterpart of any first-order theory is a conservative extension of that theory.

We observe that all the usual conditions hold for the derivability relation as defined above and that in particular the deduction theorem holds. As usual, we understand a second-order theory $T$ to be consistent (relative to this derivability relation) if there is no wff $\psi$ such that $\overline{T !} \sim(\psi \rightarrow \psi)$, and to be maximally consistent if it is consistent and for every (second-order) wff $\varphi$ of the language of $T$, if $T \cup\{\varphi\}$ is consistent, then $\varphi \in T$. We say, moreover, that $T$ is substitutionally $\omega$-complete if for all wffs $\varphi$ of the language of $T$ and for all $n \in \omega$, all $n$-place predicate variables $F^{n}$, and all individual variables $x$ :

1. $\bar{T}_{T !}(\forall x) \varphi$ iff for every individual variable $y$ that can be properly substituted for $x$ in $\varphi, \overline{T !} \varphi(y / x)$; and

2. $\bar{T}_{T}\left(\forall F^{n}\right) \varphi$ iff for every first-order wff $\psi$ of the language of $T$ and all pairwise distinct individual variables $y_{1}, \ldots, y_{n}, \leftarrow_{T !} \breve{\mho}_{\psi}^{F\left(y_{1}, \ldots, y_{n}\right)} \varphi \mid$.

We note that as is appropriate of a nominalistic theory of predication, substitutional $\omega$-completeness pertains only to the substitution of first-order wffs and not to wffs in general in the case of bound predicate variables. Despite this restriction, the following theorem is easily seen to be provable in the usual manner.

Theorem If $T$ is a predicative second-order theory and $\varphi$ is a wff of the language of $T$, then $\hbar_{T !} \varphi$ iff $\varphi$ belongs to every maximally consistent, substitutionally $\omega$-complete predicative second-order theory $S$ such that $T \subseteq S$.

(The proof of the left to right direction is of course trivial. If $\chi_{T !} \varphi$ then $T \cup\{\sim \varphi\}$ is a consistent second-order theory which can be extended in the usual way to a substitutionally $\omega$-complete theory (with infinitely many new predicate constants) which in turn, by Lindenbaum's lemma, can be extended to a maximally consistent theory which contains $T$ but to which $\varphi$ does not belong since $\sim \varphi$ does; and therefore the right to left direction is provable since its contrapositive is.) It might be noted, however, that in the initial extension to a substitutionally $\omega$-complete theory we can assume that infinitely many individual variables do not occur in the axioms of $T$ (since otherwise these axioms can be rewritten to equivalent axioms using only individual variables indexed, say, by even numbers in some enumeration of these variables). This allows us to avoid introducing individual constants, which are not required anyway in clause 1 of our definition of a substitutionally $\omega$-complete secondorder theory. The same strategy does not suffice for predicate variables, however, since unlike individual variables these variables do not occur in first-order wffs; and it is first-order wffs, not wffs in general, that are required in clause 2 of our definition. Thus we cannot avoid the introduction of (potentially) infinitely many new predicate constants occurring in axioms of the form $\left(\exists F^{n}\right) \psi \rightarrow \psi\left(P^{n} / F^{n}\right)$.

5 Nominalistic semantics for predicative second-order logic We turn now to our nominalistically motivated model-theoretic semantics for standard 
predicative second-order logic. We retain and presuppose all the semantical notions already introduced for first-order theories but give them new applications in what is to follow.

In particular, where $L$ is a language and $\mathscr{R}$ is an $L$-model, we say that $A$ is a (second-order) nominalistic assignment in 2 . if $A$ is a function with the set of individual and predicate variables as domain and such that (1) $A$, relative to the individual variables in its domain, is a first-order referential assignment in 2l., and (2) for all $n \in \omega$ and all $n$-place predicate variables $F^{n}, A\left(F^{n}\right)=$ $\left\langle x_{1}, \ldots, x_{n}, \varphi\right\rangle$, for some first-order wff $\varphi$ of $L$ and pairwise distinct individual variables $x_{1}, \ldots, x_{n}$ occurring free in $\varphi .^{9}$ Thus free predicate variables, under a nominalistic interpretation, are in effect understood to be dummy schema letters representing arbitrary predicate expressions (open wffs) of the language in question. Bound predicate variables will be similarly interpreted in our recursive definition of satisfaction. In order to do so, however, we first require the notion of the rank of a (second-order) wff: $r k(\varphi)=$ the number of occurrences of predicate quantifiers, i.e., expressions of the form $(\forall \alpha)$, where $\alpha$ is a predicate variable, occurring in $\varphi$. Where $r k(\varphi)=0$ we say that $\varphi$ is a predicative wff. It should be noted, however, that while every first-order wff is a predicative wff, not every predicative wff is a first-order wff.

Suppose $L$ is a language, $\mathfrak{A}=\langle D, f\rangle$ is an $L$-model, $A$ is a (second-order) nominalistic assignment in $\mathscr{A}$, and $k \epsilon \omega$. Then we define the satisfaction in $\mathscr{U}$ by $A$ of $a$ (second-order) wff $\varphi$ of $L$ of rank $\leqslant k$, in symbols $2 \frac{k}{A} \varphi$, by the following double recursion on the structure and rank of the wffs of $L$ :

I. if $\varphi$ is of rank $\leqslant 0$, then:

i. if $\varphi$ is $(x=y)$, then $\stackrel{0}{\stackrel{0}{A}} \varphi$ iff $A(x)=A(y)$

ii. if $\varphi$ is $P^{n}\left(x_{1}, \ldots, x_{n}\right)$, then $\mathfrak{2} \frac{0}{A} \varphi$ iff $\left\langle\left(A\left(x_{1}\right), \ldots, A\left(x_{n}\right)\right\rangle \in f\left(P^{n}\right)\right.$

iii. if $\varphi$ is $F^{n}\left(x_{1}, \ldots, x_{n}\right)$, then $2 \stackrel{\frac{0}{A}}{\frac{0}{A}} \varphi$ iff $A$ satisfies $\psi\left(x_{1} / y_{1}, \ldots, x_{n} / y_{n}\right)$ in $\mathscr{A}$, where $A\left(F^{n}\right)=\left\langle y_{1}, \ldots, y_{n}, \psi\right\rangle$

iv. if $\varphi$ is $\sim \chi$, then $2 \frac{0}{\frac{0}{A}} \varphi$ iff not $\left(\Re \frac{0}{A} \chi\right.$ )

v. if $\varphi$ is $(\psi \rightarrow \chi)$, then $2 \frac{0}{\frac{0}{A}} \varphi$ iff either not $\left(\mathfrak{A} \frac{0}{\frac{0}{A}} \psi\right)$ or $2 \frac{0}{\frac{0}{A}} \chi$

vi. if $\varphi$ is $(\forall x) \chi$, then $2 \stackrel{0}{\frac{0}{A}} \varphi$ iff for all $d \in D, \mathfrak{A} \frac{{ }_{A(d / x)}}{\bar{A}} \chi$ and

II. if $\varphi$ is of rank $\leqslant k+1$, then: repeat clauses $\mathrm{i}$-vi as above, except for replacing “थ $\frac{0}{\bar{A}}$ ” by “ $2 \frac{k+1}{{ }_{A}}$ ”, throughout; and vii. if $\varphi$ is $\left(\forall G^{m}\right) \chi$, then $2 \frac{k+1}{\frac{k+1}{A}} \varphi$ iff for all first-order wffs $\psi$ of $L$ and all pairwise distinct individual variables $x_{1}, \ldots, x_{m}$ occurring free in $\psi$,

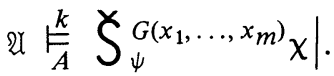

It should be noted that the notion of satisfaction involved in the definiens of clause iii of both I and II is that which has already been defined in our semantics for first-order theories. Moreover, since every first-order wff is of rank 0 , it is clear that the above definition of satisfaction for wffs of rank $\leqslant 0$ 
coincides with our original definition of satisfaction when it is restricted to first-order wffs. That is, we have the following lemma as an immediate consequence of the definition.

Lemma If $L$ is a language, $\mathfrak{O}$ is an L-model, $A$ is a nominalistic assignment in $\mathfrak{U}$, and $\varphi$ is a first-order wff of $L$, then $\mathfrak{U} \frac{0}{\frac{0}{A}} \varphi$ iff $A$ satisfies $\varphi$ in $\mathfrak{U}$.

Another immediate consequence of the definition is the fact that the satisfaction of a (second-order) wff $\varphi$ of rank $\leqslant k$ reduces to the satisfaction of $\varphi$ at its own rank.

Lemma If $L$ is a language, $\mathscr{O}$ is an L-model, $A$ is a nominalistic assignment in $\mathfrak{X}$, and $\varphi$ is any (second-order) wff of $L$ of rank $\leqslant k$, then $\mathcal{A} \frac{k}{A} \varphi$ iff $\mathscr{A} \stackrel{r k(\varphi)}{=} \varphi$.

In regard then to the nominalistic interpretation of second-order logical consequence, we say that a (second-order) wff $\varphi$ is a nominalistic (secondorder) logical consequence of a set of (second-order) wffs $T$, in symbols $T \longdiv { \overline { \text { nom } } } \varphi$, iff for every language $L$ such that $T \cup\{\varphi\}$ is a set of wffs of $L$ and for every $L$-model $\mathfrak{A}$ and nominalistic assignment $A$ in $\mathfrak{A}$, if $\mathscr{A} \stackrel{r k(\psi)}{\frac{r k}{A}} \psi$, for all $\psi \in T$, then $\mathscr{R} \frac{r k(\varphi)}{\digamma_{A}} \varphi$. Of course, where $T$ is infinite it might be that for each $k \in \omega$ there is a wff $\psi$ in $T$ such that $r k(\psi)=k$. Nevertheless, since no single wff is of an infinite rank, the above lemma allows us to bypass infinite ranks altogether in the definition of nominalistic second-order logical consequence. We say, finally, that a (second-order) wff $\varphi$ is nominalistically valid iff $\varphi$ is a nominalistic second-order logical consequence of the empty set, i.e., iff for any language $L$ of which $\varphi$ is a wff and for any $L$-model $\mathscr{A}$ and any nominalistic

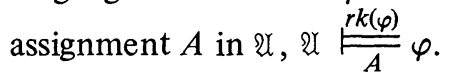

One immediate consequence of these definitions and the above lemmas is that when restricted to first-order wffs, the second-order semantical relation of logical consequence and second-order semantical property of nominalistic validity reduce to their first-order counterparts, which of course is as it should be in nominalistic semantics.

Lemma If $T \cup\{\varphi\}$ is a set of first-order $w f f$, then:

(1) $T \rightleftharpoons$

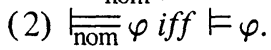

In turning now to the question of the soundness of our logical axioms, we note that except for the comprehension principle all of the remaining logical axioms are easily seen to be nominalistically valid. In regard to the comprehension principle we note first that by clause vii of part II of the definition of satisfaction the following is easily seen to be a nominalistically valid schema:

$$
\left(\forall F^{n}\right) \psi \rightarrow \stackrel{\Upsilon}{S}_{\varphi}\left(x_{1}, \ldots, x_{n}\right) \psi \mid
$$

where $\varphi$ is a first-order $w f f$ and $x_{1}, \ldots, x_{n}$ are pairwise distinct individual variables occurring free in $\varphi$. Consequently, since $F^{n}$ does not occur in $\varphi$, 
by this schema we have

$$
\left(\forall F^{n}\right) \sim\left(\forall x_{1}\right) \ldots\left(\forall x_{n}\right)\left[F\left(x_{1}, \ldots, x_{n}\right) \leftrightarrow \varphi\right] \rightarrow \sim\left(\forall x_{1}\right) \ldots\left(\forall x_{n}\right)[\varphi \leftrightarrow \varphi]
$$

and therefore

$$
\left(\exists F^{n}\right)\left(\forall x_{1}\right) \ldots\left(\forall x_{n}\right)\left[F\left(x_{1}, \ldots, x_{n}\right) \leftrightarrow \varphi\right]
$$

as nominalistically valid, where $\varphi, x_{1}, \ldots, x_{n}$ are as above.

This is not quite the comprehension principle in its original form, however, since in its original statement $\varphi$ was not required to be a first-order wff but only a wff in which no predicate variable has a bound occurrence, i.e., $\varphi$ was only required to be a predicative wff. Nevertheless, the above restricted form suffices for the validation of the original statement. To show this, suppose $F_{1}^{n_{1}}, \ldots, F_{k}^{n_{k}}$ are all the predicate variables occurring free in a wff $\varphi$ of a language $L$. Where $A$ is a nominalistic assignment in some $L$-model and

$$
\begin{gathered}
A\left(F_{1}^{n_{1}}\right)=\left\langle x_{1}, \ldots, x_{n_{1}}, \psi_{1}\right\rangle \\
\vdots \\
A\left(\dot{F}_{k}^{n_{k}}\right)=\left\langle x_{1}, \ldots, x_{n_{k}}, \psi_{k}\right\rangle
\end{gathered}
$$

we adopt the following abbreviation:

$$
A[\varphi]={ }_{d f} \breve{S}_{\psi_{1}}^{F_{1}^{n_{1}\left(x_{1}, \ldots, x_{n_{1}}\right)} \ldots \breve{S}_{\psi_{k}}^{F_{k}} k_{k}\left(x_{1}, \ldots, x_{n_{k}}\right)} \varphi|\ldots| .
$$

Lemma If $L$ is a language, $\mathfrak{Q}=\langle D, f\rangle$ is an L-model, $A$ is a (second-order) nominalistic assignment in $\mathfrak{A}$, and $\varphi$ is a wff of $L$ of rank $\leqslant k$, then $\mathfrak{A} \underset{A}{\stackrel{k}{*}} \varphi$ iff थ $\stackrel{k}{\stackrel{k}{A}} A[\varphi]$.

Proof: Suppose $L$ is a language and that for $n \in \omega, \Gamma_{n}=\{\varphi: \varphi$ is a wff of $L$ of rank $\leqslant n$ and for all $L$-models $\mathscr{U}$ and nominalistic assignments $A$ in $\mathfrak{A}, \mathfrak{N} \frac{n}{A} \varphi$ iff $\left.2 \frac{n}{\stackrel{n}{A}} A[\varphi]\right\}$. It suffices to show by strong induction that if for every $k<n$, every wff of $L$ of rank $\leqslant k$ is in $\Gamma_{k}$, then every wff of $L$ of rank $\leqslant n$ is in $\Gamma_{n}$. Assume then that for every $k<n$, every wff of $L$ of rank $\leqslant k$ is in $\Gamma_{k}$. We now show that a wff $\varphi$ of $L$ of rank $\leqslant n$ is in $\Gamma_{n}$ by induction on the subwffs of $\varphi$. If $\varphi$ is an atomic wff of the form $(x=y)$ or $P^{n}\left(x_{1}, \ldots, x_{n}\right)$, then $\varphi \in \Gamma_{n}$, since $A[\varphi]=\varphi$. If $\varphi$ is an atomic wff of the form $F^{n}\left(x_{1}, \ldots, x_{n}\right)$, then $\varphi \in \Gamma_{n}$ by clause iii of the definition of satisfaction. Finally, since:

$$
\begin{aligned}
A[\sim \psi] & =\sim A[\psi] \\
A[(\psi \rightarrow \chi)] & =(A[\psi] \rightarrow A[\chi]) \\
A[(\forall x) \psi] & =(\forall x) A[\psi] \\
A\left[\left(\forall G^{k}\right) \psi\right] & =\left(\forall G^{k}\right) A[\psi]
\end{aligned}
$$

then, by clauses iv-vii of the definition of satisfaction, it follows by the inductive hypothesis on the subwffs of $\varphi$ and by the strong inductive hypothesis on the wffs of rank $<n$ that $\varphi \in \Gamma_{n}$. 
Consider now any instance of the comprehension principle:

$$
\left(\exists F^{n}\right)\left(\forall x_{1}\right) \ldots\left(\forall x_{n}\right)\left(F\left(x_{1}, \ldots, x_{n}\right) \leftrightarrow \varphi\right),
$$

where $\varphi$ is a predicative wff of any language $L, x_{1}, \ldots, x_{n}$ are pairwise distinct individual variables occurring free in $\varphi$ and $F^{n}$ does not occur in $\varphi$. Then where $\mathfrak{Q}$ is any $L$-model and $A$ any nominalistic assignment in $\mathscr{U}$,

$$
A\left[\left(\exists F^{n}\right)\left(\forall x_{1}\right) \ldots\left(\forall x_{n}\right)\left(F\left(x_{1}, \ldots, x_{n}\right) \leftrightarrow \varphi\right)\right]
$$

reduces by definition to:

$$
\left(\exists F^{n}\right)\left(\forall x_{1}\right) \ldots\left(\forall x_{n}\right)\left(F\left(x_{1}, \ldots, x_{n}\right) \leftrightarrow A[\varphi]\right) .
$$

We note, moreover, that by definition $A[\varphi]$ is a first-order wff of $L$ since $\varphi$ is a predicative wff of $L$. Therefore, by the above lemma and the validation of the comprehension principle when restricted to first-order wffs, it follows that the comprehension principle in its full-fledged form is also nominalistically valid.

Soundness If $T$ is a predicative second-order theory and $\varphi$ is a (secondorder) $w$ ff of the language of $T$, then $\bar{T}_{T} \varphi$ only if $\varphi$ is a nominalistic (secondorder) logical consequence of $T$, i.e., only if $T \overline{\overline{\text { nom }}} \varphi$.

Proof: Suppose $\varphi$ is the terminal wff of a sequence $\Delta_{0}, \ldots, \Delta_{n}$ of wffs of the language of $T$ every constituent of which is either a member of $T$, a logical axiom, or is obtained from preceding constituents by modus ponens. Suppose, furthermore, that $L$ is a language such that $T \cup\{\varphi\}$ is a set of wffs of $L$, that $\mathscr{A}$ is an $L$-model, and that $A$ is a nominalistic assignment in 2 such that $2 \frac{r k(\psi)}{A} \psi$, for all $\psi \in T$. It suffices then to show that $2 \stackrel{r k\left(\Delta_{i}\right)}{=} \Delta_{i}$, for all $i \leqslant n$. But, by assumption, this condition holds if $\Delta_{i}$ is a member of $T$, and it also holds if $\Delta_{i}$ is a logical axiom since every logical axiom is nominalistically valid. Finally, since $r k\left(\Delta_{j} \rightarrow \Delta_{i}\right)=r k\left(\Delta_{j}\right)+r k\left(\Delta_{i}\right)$, then $r k\left(\Delta_{i}\right) \leqslant r k\left(\Delta_{j} \rightarrow \Delta_{i}\right)$; and therefore if the condition already holds for $\Delta_{j}$ and $\left(\Delta_{j} \rightarrow \Delta_{i}\right)$ as preceding constituents, then by clause $v$ of the definition of satisfaction and the second lemma of this section, the condition also holds for $\Delta_{i}$.

QED

Completeness theorem If $T$ is a predicative second-order theory and $\varphi$ is a (second-order) wff of the language of $T$, then $\bar{T}_{T !} \varphi$ iff $T \overline{\overline{\text { nom }}} \varphi$.

Proof: The left-to-right direction is of course already proved in the soundness theorem. Suppose then that $T \longdiv { \text { nom } } \varphi$ and that $S$ is a maximally consistent, substitutionally $\omega$-complete predicative second-order theory such that $T \subseteq S$. By the theorem of Section 4, it suffices to show that $\varphi \in S$.

Let $L$ be the language of $S$. We proceed as in the usual Henkin-style proof to construct an $L$-model $\mathscr{U}$ and a nominalistic assignment $A$ in $\mathscr{U}$ such that for all $k \in \omega$ and all wffs $\psi$ of $L$ of rank $\leqslant k, \psi \in S$, iff $2 \underset{A}{\frac{k}{*}} \psi$. Then, since $T \subseteq S$ and $T \overline{\overline{\text { nom }}} \varphi$, it follows that $\varphi \in S$.

Accordingly, for each individual variable $x$, let $[x]=\{y:(x=y) \in S\}$ and let $D=\{[x]: x$ is an individual variable $\}$. Also, let $f$ be a function with $L$ as 
domain and such that for all $P^{n} \in L, f\left(P^{n}\right)=\left\{\left\langle\left[x_{1}\right], \ldots,\left[x_{n}\right]\right\rangle: P^{n}\left(x_{1}, \ldots, x_{n}\right)\right.$ $\epsilon S\}$; and finally set $\mathfrak{Q}=\langle D, f\rangle$.

Toward constructing the appropriate nominalistic assignment $A$ in 2 , let us first observe that by the comprehension principle and the fact that $S$ is maximally consistent,

$$
\left(\exists G^{n}\right)\left(\forall x_{1}\right) \ldots\left(\forall x_{n}\right)\left[G\left(x_{1}, \ldots, x_{n}\right) \leftrightarrow F\left(x_{1}, \ldots, x_{n}\right)\right]
$$

belongs to $S$, for all $n \in \omega$, all pairwise distinct individual variables $x_{1}, \ldots, x_{n}$, and all $n$-place predicate variables $F^{n}$. Accordingly, since $S$ is substitutionally $\omega$-complete, there is at least one first-order wff $\psi$ such that

$$
\breve{S}_{\psi}^{G\left(x_{1}, \ldots, x_{n}\right)} \quad\left(\forall x_{1}\right) \ldots\left(\forall x_{n}\right)\left[G\left(x_{1}, \ldots, x_{n}\right) \leftrightarrow F\left(x_{1}, \ldots, x_{n}\right)\right] \mid
$$

belongs to $S$, i.e., such that

$$
\left(\forall x_{1}\right) \ldots\left(\forall x_{n}\right)\left[\psi \leftrightarrow F\left(x_{1}, \ldots, x_{n}\right)\right]
$$

belongs to $S$. Assuming then some readily constructible well-ordering of the first-order wffs of $L$ and thereby also a well-ordering, for each $n \in \omega$, of all $(n+1)$-tuples $\left\langle x_{1}, \ldots, x_{n}, \psi\right\rangle$, where $\psi$ is a first-order wff of $L$ and $x_{1}, \ldots, x_{n}$ are pairwise distinct individual variables occurring free in $\psi$, we set $A\left(F^{n}\right)=$ $\left\langle x_{1}, \ldots, x_{n}, \psi\right\rangle$, where this is the first $(n+1)$-tuple of this form to satisfy the above condition with respect to $F^{n}$. In addition, for each individual variable $x$, we set $A(x)=[x]$.

Let us observe at this point that if $\psi$ is a first-order wff of $L$, then, as in the completeness proof for first-order theories, we can show by a simple induction on the number of logical constants occurring in $\psi$ that $\psi \in S$ iff $A$ satisfies $\psi$ in $\mathscr{A}$. We do not bother to repeat the proof here but assume it in what follows.

We now show by strong induction that for all $k \in \omega$ and all wffs $\psi$ of $L$ of rank $\leqslant k, \psi \in S$ iff $\mathfrak{a} \underset{A}{\frac{k}{*}} \psi$. Suppose then that for all $m<k$ and every wff $\psi$ of $L$ of rank $\leqslant m, \psi \in S$ iff $2 \frac{m}{\frac{m}{A}} \psi$. We proceed to show by a second induction on the number of logical constants occurring in the wffs $\chi$ of $L$ of rank $\leqslant k$ that $\chi \in S$ iff $2 \frac{k}{\frac{k}{A}} \chi$. Suppose then that $\chi$ is a wff of $L$ of rank $\leqslant k$. If $\chi$ is an atomic wff either of the form $(x=y)$ or $P^{n}\left(x_{1}, \ldots, x_{n}\right)$, then since $(x=y) \in S$ iff $[x]=[y]$, and since $P^{n}\left(x_{1}, \ldots, x_{n}\right) \in S$ iff $\left\langle\left[x_{1}\right], \ldots,\left[x_{n}\right]\right\rangle \in f\left(P^{n}\right)$, then by the definitions of $A$ and $f$, it follows that $\chi \in S$ iff $2 \underset{A}{k} \chi$. On the other hand, if $\chi$ is an atomic wff of the form $F^{n}\left(x_{1}, \ldots, x_{n}\right)$, then since

$$
\left(\forall x_{1}\right) \ldots\left(\forall x_{n}\right)\left[\psi \leftrightarrow F\left(x_{1}, \ldots, x_{n}\right)\right]
$$

belongs to $S$, where $A\left(F^{n}\right)=\left\langle x_{1}, \ldots, x_{n}, \psi\right\rangle$, etc., then $F^{n}\left(x_{1}, \ldots, x_{n}\right) \in S$ iff $\psi \in S$; and since, as noted above, $\psi \in S$ iff $A$ satisfies $\psi$ in $\mathscr{A}$, it follows by clause iii of the definition of satisfaction that $F^{n}\left(x_{1}, \ldots, x_{n}\right) \in S$ iff $2 \underset{A}{\frac{k}{A}}$ $F^{n}\left(x_{1}, \ldots, x_{n}\right)$. If $\chi$ is of the form $\sim \chi,\left(\psi_{1} \rightarrow \psi_{2}\right)$ or $(\forall x) \psi$, then by the inductive hypothesis and essentially the same argument as in the completeness proof 
for first-order theories, it follows that $\chi \in S$ iff $\mathscr{\alpha} \frac{k}{\frac{k}{A}} \chi$. Finally, if $\chi$ is of the form $\left(\forall F^{n}\right) \psi$, then $k=m+1$, for some $m \in \omega$, and therefore by clause vii of the definition of satisfaction, $\mathfrak{A} \underset{A}{\frac{k}{F}}\left(\forall F^{n}\right) \psi$ iff for all first-order wffs $\varphi$ of $L$ and pairwise distinct individual variables $x_{1}, \ldots, x_{n}, \mathfrak{A} \underset{A}{\stackrel{m}{F}} \zeta_{\varphi} F\left(x_{1}, \ldots, x_{n}\right) \psi \mid$. But, by the (strong) inductive hypothesis,

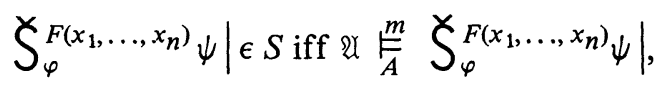

for all first-order wffs $\varphi$ of $L$; and therefore, since $S$ is substitutionally $\omega$ complete, $\left(\forall F^{n}\right) \psi \in S$ iff $2 \frac{k}{A}\left(\forall F^{n}\right) \psi$.

QED

As an immediate corollary of the above completeness theorem, together with the completeness theorem for first-order theories and the third of the above lemmas, it follows that the predicative second-order counterpart of any first-order theory is always a conservative extension of that theory.

Corollary If $T$ is a first-order theory and $\varphi$ is a first-order wff of the language of $T$, then $\hbar_{T !} \varphi$ iff $\hbar_{T} \varphi$.

6 A nonstandard predicative second-order logic We have referred throughout this paper to standard predicative second-order logic so as to distinguish it from a certain nonstandard version formulated in an earlier paper and to which our nominalistic semantics does not apply (cf. [3]). Indeed, it is not a nominalist theory of predication which this alternative version was designed to represent but a form of conceptualism according to which there are intelligible universals, i.e., ( $n$-ary) concepts over and above ( $n$-place) predicate expressions but not such as can exist independently of both language and thought, and in particular not such as can be specified only by impredicative wffs, i.e., wffs in which predicate variables have bound occurrences.

The intelligible universals or concepts of this form of conceptualism are understood to be cognitive capacities for classifying, characterizing, and relating individuals in various ways; and as such they are neither images nor particular mental occurrences but are rather unsaturated cognitive structures whose realization in thought is what informs particular mental acts with a predicable nature. Quantificational reference to such universals is accordingly not a restricted form of quantificational reference to individuals but is rather sui generis and incomparable with any notion of reference that applies to individuals. It is because the universals of this form of conceptualism can exist only relative to the power of the intellect to form or construct them that the appropriate logical medium in which such a quantificational reference is to be represented is a form of predicative second-order logic. Accordingly, since it will help elucidate the sense in which standard predicative second-order logic represents a nominalist theory of predication, we shall conclude this paper with a brief comparison of these two frameworks and their respective logics.

Both frameworks, it should be noted, are based on the same logical grammar and, except for the comprehension principle, the same logical axioms. Their point of departure is in their construal of the difference between free and 
bound predicate variables and in the interpretation which each allows to be given to the identity sign and to predicate constants. In particular, the comprehension principle of our nonstandard version has the following form:

CP! $\quad\left(\forall G_{1}^{k_{1}}\right) \ldots\left(\forall G_{i}^{k_{i}}\right)\left(\exists F^{n}\right)\left(\forall x_{1}\right) \ldots\left(\forall x_{n}\right)\left[F\left(x_{1}, \ldots, x_{n}\right) \leftrightarrow \varphi\right]$

where $\varphi$ is a pure second-order wff in which no predicate constants or the identity sign occur and in which no predicate variable has a bound occurrence, i.e., $\varphi$ is a predicative wff, $G_{1}^{k_{1}}, \ldots, G_{i}^{k_{i}}$ are all of the predicate variables occurring (free) in $\varphi$, and $x_{1}, \ldots, x_{n}$ are pairwise distinct individual variables occurring free in $\varphi$.

We observe that $L L_{2}$, our second-order analogue of Leibniz's law, remains provable just as in standard predicative second-order logic. Nevertheless, because of the form of the above comprehension principle, the second-order principle of universal instantiation is now provable only in the following forms:

$$
\begin{array}{ll}
\text { UI! } \quad\left(\exists F^{n}\right)\left(\forall x_{1}\right) \ldots\left(\forall x_{n}\right)\left[F\left(x_{1}, \ldots, x_{n}\right) \leftrightarrow \varphi\right] \rightarrow\left[\left(\forall G^{n}\right) \psi \rightarrow \breve{S}_{\varphi}^{G\left(x_{1}, \ldots, x_{n}\right)} \psi \mid\right] \\
\text { UI! } \quad\left(\forall G_{1}^{k_{1}}\right) \ldots\left(\forall G_{i}^{k_{i}}\right)\left[\left(\forall F^{n}\right) \psi \rightarrow \breve{S}_{\varphi}^{F\left(x_{1}, \ldots, x_{n}\right)} \psi \mid\right]
\end{array}
$$

where $\varphi, F^{n}, G_{1}^{k}, \ldots, G_{i}^{k_{i}}, x_{1}, \ldots, x_{n}$ are as described in CP!. The point here, as in standard predicative second-order logic, is that an open wff $\varphi$ can be taken as specifying a predicative concept only if all of the predicates occurring in it can also be taken as specifying predicative concepts, but, and here is where the system departs from standard predicative second-order logic, it is also allowed here that free predicate variables, and the identity stgn and predicate constants as well, might not themselves have predicative concepts as their semantical counterparts-though in that case, strictly speaking, they will not have any semantical counterparts at all but will only be, as it were, syncategorematic signs of the system. (What we have here, in other words, is a variation on the idea of a "free logic", except now applied to predicate variables and constants rather than to individual variables and constants.)

Nominalists of course have themselves construed predicate constants as syncategorematic signs (cf. [5], p. 105). And the fact that we introduced predicate quantifiers into the nominalist framework did not really change their status from syncategorematic to categorematic signs, since these quantifiers were not interpreted referentially but only substitutionally. But then it is essential to this interpretation that the significance which predicate quantifiers have in a given applied theory is completely determined by what predicate constants, and thereby what first-order wffs, are present in that theory.

Our constructive conceptualist agrees that some predicate constants may indeed be only syncategorematic signs, but he disagrees that all must be so construed. Indeed, whether an $n$-place predicate constant $P^{n}$ of the language of an applied theory $T$ is construed in $T$ as a syncategorematic or a categorematic sign will depend on whether

$$
\left(\exists F^{n}\right)\left(\forall x_{1}\right) \ldots\left(\forall x_{n}\right)\left[F\left(x_{1}, \ldots, x_{n}\right) \leftrightarrow P\left(x_{1}, \ldots, x_{n}\right)\right]
$$

is provable in $T$ (whether because it is a "meaning postulate" of $T$ or otherwise 
derived from such postulates for other predicate constants and the axioms of $T$ ), since only then, by UI!, will $P^{n}$ be a legitimate substituend of the bound $n$-place predicate variables of $T$. That is, instead of the significance which predicate quantifiers have in a given applied theory being determined by what predicate constants, and thereby what first-order wffs, are present in that theory, it is in fact their conceptually prior and independent referential significance which determines whether a predicate constant is a categorematic sign standing for a predicative (constructible) concept or a syncategorematic sign which is not an allowable substituend of any bound predicate variable and whose significance in that respect is completely immanent to the theory in question.

Now it is not an arbitrary decision whether a predicate constant stands for a predicative (constructible) concept or not, but will in general depend on the role assigned the predicate constant in the (nonstandard) predicative second-order theory in question. Ordinarily, it is only when the description of that role in the theory itself is essentially impredicative that the predicate constant will be construed as syncategorematic; and even then it will usually be provable in the theory that the predicate constant must be a syncategorematic sign, i.e., that it cannot, on pain of contradiction, be a legitimate substituend of any bound predicate variable.

Consider, for example, a predicative second-order theory regarding membership, represented by the two-place predicate constant ' $\epsilon$ ', in which it is claimed that there exists a set corresponding to any predicative (constructible) concept:

$$
(\forall F)(\exists y)(\forall x)[x \in y \leftrightarrow F(x)] .
$$

By Russell's argument, such a claim in standard predicative second-order logic is clearly inconsistent, since in that framework ' $x \notin x$ ' is a legitimate substituend of the bound monadic predicate variables. In the nonstandard predicative second-order logic, on the other hand, this claim is both consistent and plausible, and all that follows by Russell's argument is that ' $\epsilon$ ' cannot stand for a predicative (binary) concept. And that in a way is as it should be since the significance which ' $\epsilon$ ' is portrayed as having in this second-order theory is clearly impredicative.

Thus, in general, while the pure second-order wff (where $R$ is a two-place predicate variable):

$$
(\forall F)(\exists y)(\forall x)[R(x, y) \leftrightarrow F(x)]
$$

is consistent in the referential semantics of the nonstandard predicative secondorder logic, the same wff is inconsistent in the nominalistic semantics of standard predicative second-order logic. This is not to say, however, that one is the right and the other the wrong semantics (unless of course one is adopting a metaphysical, rather than merely a logical, stance). It is only to point out that the theories of predication which the two systems and their respective semantics represent are motivated on different philosophical grounds. For the nominalist, predicate quantifiers have no referential significance, there being no universals beyond predicate expressions themselves; 
and therefore all predicates are in this sense syncategorematic signs for the nominalist. For the conceptualist, however, predicate quantifiers do have referential significance and refer in fact to concepts, i.e., certain cognitive capacities whose mode of existence is a functional expression of the powers of the intellect and which therefore are not themselves independently existing individuals. (Nor for that matter are they individuals in any other sense as well.) And it is because predicate quantifiers have such a referential significance for the conceptualist rather than the merely substitutional interpretation of the nominalist that new predicate expressions are generated which cannot themselves stand for a concept which it is within the power of the human intellect to form or construct independently of the theoretical nexus in which such predicate quantifiers occur. These additional contexts or predicate expressions are well-formed within that theoretical nexus, but since they cannot be substituends of the bound predicate variables, they must accordingly be construed as purely syncategorematic signs of the theory in question. ${ }^{10}$

It is, moreover, because identity allows for full substitutivity with respect to these syncategorematic as well as the categorematic predicate expressions that we have excluded it from occurring in the comprehension principle $\mathrm{CP}$ ! of the nonstandard logic, since in that case it will exceed the bounds of predicativity. In this regard, while both nominalism and conceptualism agree that any given applied second-order theory will contain at most finitely many predicate constants-though of course for both there are potentially infinitely many predicate constants that may be introduced in the development of that theory or any of its definitional extensions-they will disagree as to whether indiscernibility with respect to these finitely many predicate constants amounts itself to a predicative wff as well as to whether such indiscernibility suffices for full substitutivity. Thus, for example, if $P^{1}$ and $Q^{2}$ are the only predicate constants of an applied theory $T$, then for the nominalist, but not for the conceptualist, the identity wff $(x=y)$ is reducible in $T$ to (cf. [8], p. 63f):

$$
[P(x) \leftrightarrow P(y)] \wedge(\forall z)[Q(x z) \leftrightarrow Q(y z)] \wedge(\forall z)[Q(z x) \leftrightarrow Q(z y)]
$$

which for the nominalist, but not always for the conceptualist, is a predicative wff of $T$. It is a predicative wff of $T$ for the conceptualist only if both $P^{1}$ and $Q^{2}$ are categorematic signs in $T$, i.e., only if

$$
\begin{aligned}
& \left(\exists F^{1}\right)(\forall x)[F(x) \leftrightarrow P(x)] \\
& \left(\exists R^{2}\right)(\forall x)(\forall y)[R(x, y) \leftrightarrow Q(x, y)]
\end{aligned}
$$

are provable in $T$. But even if they are, the above indiscernibility wff will still not suffice for a reduction of identity, as far as the conceptualist is concerned, since it will in general not suffice to justify full substitutivity in contexts with free predicate variables. Of course for the nominalist contexts with free predicate variables always amount to a schematic representation of firstorder wffs, and for that reason identity, for the nominalist, is reducible to indiscernibility with respect to the finitely many predicate constants available in any given theory. That is why we have allowed identity wffs to occur in the comprehension principle of standard predicative second-order logic.

Finally, as a further and final indication of the important difference in these logics of the role of predicate constants and free predicate variables we 
note that the substitution rule:

S If $\vdash \psi$, then $\vdash \dot{S}_{\varphi} F\left(x_{1}, \ldots, x_{n}\right) \psi \mid$

where $F^{n}$ is a predicate variable or constant and $\varphi$ is any second-order wff, predicative or otherwise, is a derived rule of our nonstandard predicative second-order logic and not a rule at all, derived or otherwise, of standard predicative second-order logic. Note in this last regard that since free ( $n$-place) predicate variables (representing arbitrary first-order wffs) can always be instantiated for bound ( $n$-place) predicate variables (also representing arbitrary first-order wffs), then by $\mathrm{UI}_{2}$ :

$$
\left(\forall F^{n}\right) \psi \rightarrow \psi
$$

is a theorem of standard predicative second-order logic (and not a theorem of our nonstandard alternative). But then if $S$ were added as a new rule of inference to standard predicative second-order logic, we would thereby justify the unqualified principle of universal instantiation:

$$
\left(\forall F^{n}\right) \psi \rightarrow \text { \} _ { \varphi } ^ { F ( x _ { 1 } , \ldots , x _ { n } ) } \psi}
$$

and thereby also the unqualified comprehension principle of standard impredicative second-order logic. Thus, while $S$ is a derived rule of our nonstandard predicative second-order logic, it is not only not a derived rule of standard predicative second-order logic but is completely inadmissible on nominalist grounds. Its addition to standard predicative second-order logic amounts in effect to a rejection of the nominalist framework and a nominalist theory of predication.

\section{NOTES}

1. For the consensus view, see Parsons [9]. For the dissenting view, at least in regard to the extension of predicative second-order logic to ramified type theory, see Church [2]. It is possible of course that Church intends his demurral to apply only after predicates are ramified and allowed to occur as subjects of higher-order predicates. If so, then we believe that his demurral may have some merit (see Note 10).

2. For reasons indicated in Note 10 , we suspect that ramification may presuppose a linguistic capacity for introducing predicates that exceeds the proper limits of a nominalist theory of predication. Such a capacity does not exceed the limits of a closely related form of conceptualism (briefly discussed in Section 6) which may be represented by the nonstandard predicative second-order logic formulated in [3].

3. Although some nominalists may reject the assumption of an actual, as opposed to a merely potential, infinity, others find the actual infinity of, e.g., sets or real numbers to be unproblematic. Nevertheless, for both types of nominalism the notion of an actual infinity of possible predicates as applied to the individuals of a potentially infinite domain of discourse is (or should be) problematic. For as Charles Parsons has noted, "the 'totality' of possible predicates is irremediably potential, and more radically so than the natural numbers are on the intuitionistic or other constructive conceptions, since in the former case there is no rule which 'generates' all predicates on an infinite domain, even modulo extensional equivalence” [10], p. 8. 
4. We say that $\psi$ is a generalization of $\varphi$ iff $\psi$ is $\left(\forall x_{1}\right) \ldots\left(\forall x_{n}\right) \varphi$. In case $n=0,\left(\forall x_{1}\right) \ldots$ $\left(\forall x_{n}\right)$ is assumed to be the null sequence.

5. Of course our model theory, and our syntactical metalanguage, will not be acceptable in their present form to a nominalist who rejects sets and actual infinities. We assume in this regard that the entirety of our metalanguage can in principle be reformulated or translated into an applied predicative second-order nominalistic system such as that described below for a domain of discourse in which no more than a potential infinity of expression-types need be assumed. Expression-types are of course themselves abstract entities and it is possible that they too may be eliminated in favor of expression-tokens. We ourselves, however, are unsure of the feasibility of this last reduction.

6. In case $n=0$, we take $\left(x_{1}, \ldots, x_{n}\right)$ to be the null sequence.

7. We now understand $\psi$ to be a generalization of $\varphi$ iff $\psi$ is $\left(\forall a_{1}\right) \ldots\left(\forall a_{n}\right) \varphi$, where each $a_{i}$ is either an individual or a predicate variable.

8. The requirement that the pairwise distinct individual variables $x_{1}, \ldots, x_{n}$ occur in $\varphi$ can be dropped since, e.g., $\left(\varphi \leftrightarrow \varphi \wedge\left[G\left(x_{1}, \ldots, x_{n}\right) \vee \sim G\left(x_{1}, \ldots, x_{n}\right)\right]\right)$ is tautologous.

9. The requirement that $x_{1}, \ldots, x_{n}$ occur free in $\varphi$, though natural in its motivation, can be dropped since $\varphi$ is interchangeable with $\left(\varphi \wedge x_{1}=x_{1} \wedge \ldots \wedge x_{n}=x_{n}\right)$, which is also a first-order wff of $L$.

10. They may, however, come to be considered as categorematic signs of a new stage of concept-formation which by a process of reflective abstraction may be constructed on the basis of the logical structure of predicative concepts. The concepts of this new stage will then include all the predicative concepts as well as those whose formation presupposes quantificational reference to predicative concepts. Predicate quantifiers for these new concepts will then of course generate new predicate expressions that are syncategorematic signs with respect to this stage of concept-formation, but which in turn become categorematic signs of a yet further stage and so on ad infinitum through all the potentially infinitely many stages of ramified second-order logic. We believe that the principles needed for the development of these successive stages of concept-formation can be justified within the framework of Jean Piaget's genetic epistemology. It should be noted, however, that essentially the same logical structure as our nonstandard predicative second-order logic is repeated at each of these stages with no one of them closing the gap or openness of the role of free predicate variables so as to reach a logical structure resembling standard predicative second-order logic. Indeed, it is the "nonstandard" feature of the logic at each of these stages which both suggests and leads to the construction of the next successive stage. Full closure or the conceptual overcoming of this "nonstandard" feature can be accomplished only by a radically new type of constructive abstraction resulting in standard impredicative second-order logic. (We believe, incidentally, that the principles of genetic epistemology can justify the move to such a radically new type or stage of concept-formation.)

Finally, it should be noted that none of these successive stages of conceptformation, including the controversial stage at which impredicative concept-formation becomes possible, justifies the move to third- and higher-order logic where concepts are considered in effect as higher-order types of (saturated) individuals. For the platonic realist for whom universals are independently existing individuals, such a move is unproblematic. But for the conceptualist for whom concepts are cognitive capacities and therefore never themselves individuals (of any type), such a move presupposes a stage of conceptual involvement at which predicate-nominalizations become possible and at which "concept correlates," e.g., sets or other abstract individuals, may have to finally be introduced (in a manner reminiscent of Frege). Here special caution is 
demanded since apparently plausible assumptions regarding such concept correlates easily lead to paradox (as Frege himself discovered). Cf. the author's [4].

\section{REFERENCES}

[1] Church, A., Introduction to Mathematical Logic, Princeton University Press, Princeton, N.J., 1956.

[2] Church, A., "Comparison of Russell's resolution of the semantical antinomies with that of Tarski," The Journal of Symbolic Logic, vol. 41 (1976), pp. 747-760.

[3] Cocchiarella, N., "A new formulation of predicative second order logic," Logique et Analyse, vol. 17, no. 65-66 (1974), pp. 61-88.

[4] Cocchiarella, N., "On the logic of nominalized predicates and its philosophical interpretations," Erkenntnis, vol. 13 (1978), pp. 339-369.

[5] Goodman, N. and W. V. O. Quine, "Steps toward a constructive nominalism," The Journal of Symbolic Logic, vol. 12 (1947), pp. 105-122.

[6] Kalish, D. and R. Montague, "On Tarski's formulation of predicate logic with identity," Archiv für Mathematische Logik und Grundlagenforschung, vol. 7 (1965), pp. 81-101.

[7] Leblanc, H. and G. Weaver, "Truth-functionality and the ramified theory of types," in Truth, Syntax and Modality, ed., H. Leblanc, Amsterdam, 1973, pp. 148-167.

[8] Quine, W. V. O., Philosophy of Logic, Prentice-Hall, Englewood Cliffs, N.J., 1970.

[9] Parsons, C., “A plea for substitutional quantification," The Journal of Philosophy, vol. 68 (1971), pp. 231-237.

[10] Parsons, C., "Sets and classes," Noûs, vol. 8 (1974), pp. 1-12.

[11] Tarski, A., "A simplified formulation of predicate logic with identity," Archiv für Mathematische Logik und Grundlagenforschung, vol. 7 (1965), pp. 61-79.

Department of Philosophy

Indiana University

Bloomington, Indiana 47401 